\title{
COVID-19 Scenarios: an interactive tool to explore the spread and associated morbidity and mortality of SARS-CoV-2
}

\author{
Nicholas B Noll, ${ }^{1,2}$ Ivan Aksamentov, ${ }^{1}$ Valentin Druelle, ${ }^{1}$ Abrie Badenhorst, ${ }^{3}$ Bruno Ronzani, ${ }^{3}$ Gavin Jefferies, ${ }^{3}$ \\ Jan Albert, ${ }^{4,5}$ and Richard A Neher ${ }^{1,2}$ \\ ${ }^{1}$ Biozentrum, University of Basel \\ ${ }^{2}$ Swiss Institute of Bioinformatics, Basel, Switzerland \\ ${ }^{3}$ unaffiliated contributors \\ ${ }^{4}$ Department of Clinical Microbiology, Karolinska University Hospital, Stockholm, Sweden \\ ${ }^{5}$ Department of Microbiology, Tumor and Cell Biology, Karolinska Institutet, Stockholm, Sweden
}

(Dated: May 8, 2020)

\begin{abstract}
The ongoing SARS-CoV-2 pandemic has caused large outbreaks around the world and every heavily affected community has experienced a substantial strain on the health care system and a high death toll. Communities therefore have to monitor the incidence of COVID-19 carefully and attempt to project the demand for health care. To enable such projections, we have developed an interactive web application that simulates an age-structured SEIR model with separate compartments for severely and critically ill patients. The tool allows the users to modify most parameters of the model, including age specific assumptions on severity. Infection control and mitigation measures that reduce transmission can be specified, as well as age-group specific isolation.

The simulation of the model runs entirely on the client side in the browser; all parameter settings and results of the simulation can be exported for further downstream analysis. The tool is available at covid19-scenarios.org and the source code at github.com/neherlab/covid19_scenarios
\end{abstract}

The novel coronavirus SARS-CoV-2 was first detected in the city of Wuhan within the Hubei province of China at the end of December 2019 (Li et al., 2020). In the following months, SARS-CoV-2 has shown to be highly transmissible - the basic reproductive number, $R_{0}$, has been estimated to be within 2-3 (Riou and Althaus, 2020. Zhang et al., 2020) with an estimated serial interval of 5-7 days (Ganyani et al., 2020; Nishiura et al., 2020). The basic reproduction number likely varies between communities and is affected by intervention measures. The illness caused by SARS-CoV-2 infection, COVID-19, clinically presents with a large variance of symptoms that range from mild and asymptomatic infection to acute severe respiratory illness. The clinical presentation of the infection strongly depends upon patient age (Surveillances, 2020) and certain comorbidities (Fang et al., 2020). The WHO declared the COVID-19 outbreak a pandemic on March 11th, 2020 (The WHO COVID-19 group, 2020). As of April 20th, 2020, there have been over 2.4 million confirmed COVID-19 cases from 210 countries.

A critical component of the global response to the COVID-19 pandemic is the possibility to explore different scenarios for local outbreaks within communities across the world using mathematical modelling. Modelling is important not only to guide governmental public health policy but also to inform hospital readiness and educate the general public on the importance of social distancing efforts. The spectrum of models used to analyze COVID-19 outbreaks ranges from computationally intensive agent-based simulation (Neil M Ferguson, 2020), variants of SIR/SEIR models (Kermack et al., 1927), to phenomenological curve fitting approaches "IMHE COVID-19 forecasting team" and Murray, 2020). However, traditional epidemiological modelling protocols do not scale for a global pandemic - modelling has to be done on a region-by-region basis. Thus, to make such modeling widely available, we have developed an interactive, online tool that allows users to efficiently explore COVID-19 scenarios based upon different epidemiological assumptions and potential mitigation strategies. The dynamics are modelled by an age-stratified SEIR model, with additional novel compartments that correspond to hospital and ICU utilization with finite capacity.

Our deterministic approach strikes a compromise between the accuracy of the approximation of the outbreak dynamics and the speed of the simulation. On a typical modern computer and browser, the simulation will complete in under one second such that many different parameter values can be explored interactively. The output of the model is a time series of simulated COVID-19 infections, hospitalizations, and ICU usage. Surveillance data such as case counts, COVID-19-related fatalities, and hospitalizations can be compared to the model output when such data are available. Additionally, we utilize these data to estimate a few basic parameters for each provided scenario to provide reasonable starting points for further parameter explorations. However, we stress that the focus of this tool is on the exploration of scenarios and not on parameter inference.

We have designed our tool with the following principles: (i) users should be able to interact dynamically with the simulation such that changing underlying as- 
sumptions manifests instantly in the results, (ii) empirical surveillance data should be plotted with the simulation results to allow for easy assessment of parameter assumptions, (iii) results should be easily shareable via URLs, exported raw data, and parameter files. Our tool, COVID-19 Scenarios, was first released on March 9, 2020 and was one of the first publicly available interactive models. It has been utilized consistently throughout the COVID-19 pandemic, averaging roughly 8 thousand page loads per day. Since we first released, we have been dedicated to improving the tool, in both its underlying scientific accuracy as new data emerged, as well as the overall user experience. All source code and the aggregated surveillance data are made freely available through GitHub.

\section{BASIC MODEL}

We approximate the dynamics of a COVID-19 outbreak using a generalized SEIR model in which the population is partitioned into age-stratified compartments of: susceptible (S), exposed (E), infected (I), hospitalized $(\mathrm{H})$, critical $(\mathrm{C})$, ICU overflow $(\mathrm{O})$, dead (D) and recovered (R) individuals (Kermack et al., 1927). The progression of illness is approximated by the following compartment transitions: susceptible individuals are exposed to the virus by contact with an infected individual; exposed individuals progress towards a infectious state; infectious individuals either recover without hospitalization or progress towards a severe illness that requires hospitalization; hospitalized individuals either recover or worsen towards a critical state; individuals with a critical illness either transition to the ICU or, if the hospital is at capacity, to an "overflow" compartment and either return to the hospital state or die; recovered individuals can not be infected again. See Fig. 1 for an illustration of the model. We note that direct comparisons between the model predictions and available surveillance data are difficult since only a fraction of cases are confirmed by a positive test and this fraction various between regions. The number of COVID-19 deaths is often a more robust measure.

Let $a, b \in\left[1,2, \ldots, N_{a}\right]$ denote the different age classes of each compartment. The parameters of the model fall into three broad categories: a time-dependent infection rate $\beta_{a}(t)$; the rate of transition out of the exposed, infectious, hospitalized, and critical/overflow compartments $\gamma_{e}, \gamma_{i}, \gamma_{h}$, and $\gamma_{c}$ respectively; and the age-specific fractions $m_{a}, c_{a}$ and $f_{a}$ of mild, critical, and fatal infections respectively. Below, we expound upon each class of parameter.

The rate of transmission, $\beta_{a}(t)$, is nominally determined by both the basic reproductive number $R_{0}$ and the time period of patient infectivity $\gamma_{i}^{-1}$. Additionally, the rate of transmission can be effectively slowed by mitiga- tion efforts (e.g. social distancing), which we account for phenomenologically by a multiplicative factor $\mathcal{M}(t)$ (see below). Lastly, empirical data shows a strong, consistent seasonal variation of the four endemic coronaviruses suggesting similar seasonality in the transmissibility of SARS-CoV-2 (Neher et al., 2020) Taken together, the rate of transmission is modelled by

$$
\beta_{a}(t)=\gamma_{i} R_{0} \chi_{a} \mathcal{M}(t)\left(1+\varepsilon \cos \left(2 \pi\left(t-T_{\text {max }}\right)\right)\right)
$$

where $\chi_{a}$ models specific demographic isolation, and $\varepsilon$ and $T_{\max }$ denote the (currently unknown) amplitude of seasonal variation in transmissibility and the time of the year of peak transmission respectively.

After an individual is infected (i.e. was exposed) it takes some time before the individual itself is infectious. In our model, the average value of this latency is given by $\gamma_{e}^{-1}$. The incubation time of COVID-19 has been estimated to be well approximated by an Erlang distribution (Lauer et al. 2020 ). As such, we approximate the distribution of incubation times within our framework by chaining three exposed states in which the mean time to pass through all three states is $\gamma_{e}^{-1}$. The mean infectious time of a COVID-19 case is $\gamma_{i}^{-1}$, which together with the incubation period $\gamma_{e}^{-1}$ defines the serial interval. The residence times in the remaining compartments are assumed to be exponentially distributed and thus taken to be a singular state.

As noted above, the fraction of COVID-19 infections that are asymptomatic/mild, severe cases which progress to a critical state, and critical cases that are fatal are denoted as $m_{a}, c_{a}$, and $f_{a}$, respectively (see below for more detail). However, it is important to consider the effects of hospital capacity and overutilization in forecasting potential scenarios. Finite hospital resources and staffing acutely impact the outcome for critical COVID-19 patients and thus the overall COVID-19-related fatalities. We phenomenologically capture this effect by introducing non-linear constraint of a finite number of ICU beds $\mathcal{C}$ that can accommodate critical patients. Once the number of critical cases exceeds this parameters, additional critical cases are redirected to an "overflow" compartment. We take the mortality rate of an overflow patient relative to a patient with an ICU bed to be $\xi$.

With all parameters explicitly defined, our full model 


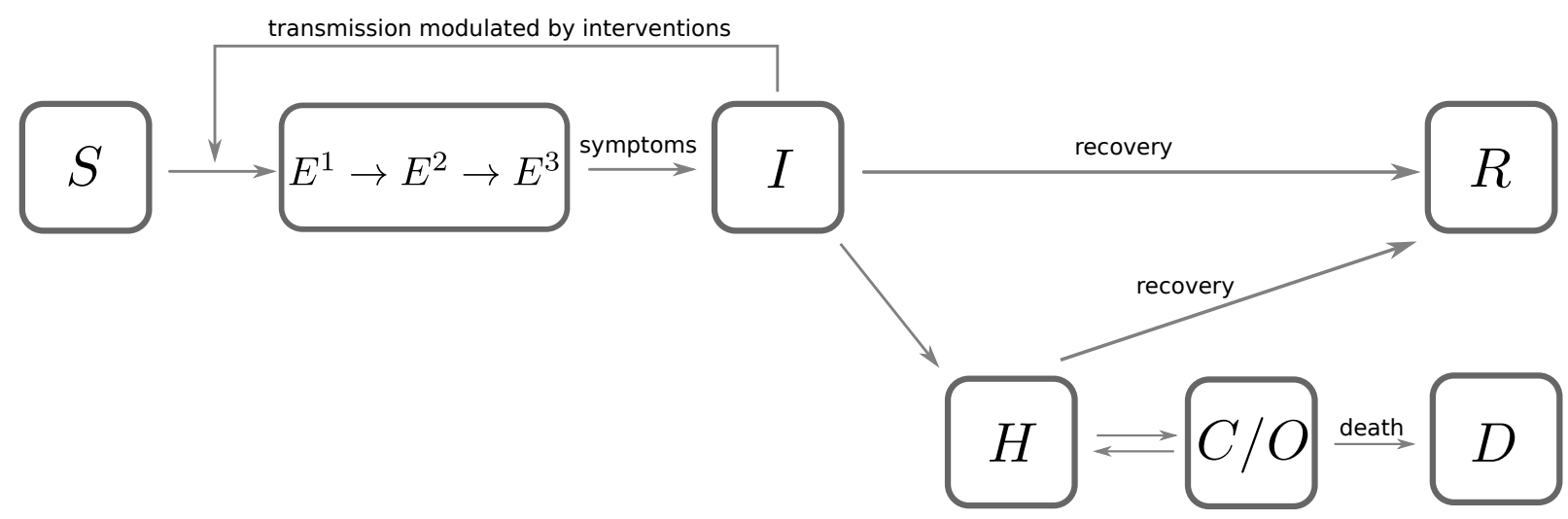

Figure 1 Diagram of model. A schematic illustration of the underlying model and allowed transitions. $S, E^{i}, I, R, H, C, O$ and $D$ represent the susceptible, exposed, infectious, recovered, hospitalized, critical, overflow, and fatal compartments of the model. Each compartment is further stratified by age demographics.

can be written

$$
\begin{aligned}
\frac{d S_{a}}{d t} & =-\beta_{a}(t) \sum_{b} S_{a} I_{b} \\
\frac{d E_{a}^{1}}{d t} & =+\beta_{a}(t) \sum_{b} S_{a} I_{b}-3 \gamma_{e} E_{a}^{1} \\
\frac{d E_{a}^{2}}{d t} & =3 \gamma_{e}\left[E_{a}^{1}-E_{a}^{2}\right] \\
\frac{d E_{a}^{3}}{d t} & =3 \gamma_{e}\left[E_{a}^{2}-E_{a}^{3}\right] \\
\frac{d I_{a}}{d t} & =3 \gamma_{e} E_{a}^{3}-\gamma_{i} I_{a} \\
\frac{d H_{a}}{d t} & =\left[1-m_{a}\right] \gamma_{i} I_{a}+\gamma_{c}\left[1-f_{a}\right]\left[C_{a}+\xi O_{a}\right]-\gamma_{h} H_{a} \\
\frac{d C_{a}}{d t} & =c_{a} \gamma_{h} \Theta\left(\mathcal{C}-C_{s}\right) H_{a}-\gamma_{c} C_{a} \\
\frac{d O_{a}}{d t} & =c_{a} \gamma_{h} \Theta\left(C_{a}-\mathcal{C}\right) H_{a}-\left[1+(\xi-1) f_{a}\right] \gamma_{c} O_{a} \\
\frac{d R_{a}}{d t} & =m_{a} \gamma_{i} I_{a}+\left[1-c_{a}\right] \gamma_{h} H_{a} \\
\frac{d D_{a}}{d t} & =f_{a} \gamma_{c}\left[C_{a}+\xi O_{a}\right]
\end{aligned}
$$

where $\Theta(x)$ denotes the Heaviside step function and imposes the finite constraint of hospital resources.

The model currently does not allow for reinfection. Evidence from other coronaviruses suggests that infection elicits immunity that lasts for at least a year (Callow et al. 1990). Whether this also holds for SARS-CoV-2 is not yet clear, but reinfection and herd immunity are of minor relevance in the early phase of a pandemic (Neher et al. 2020). Reinfection might be added to the model in the future if evidence accumulates that it is important.

\section{USER INTERFACE AND ADJUSTABLE PARAMETERS}

Epidemiological models, including the one defined by Eqn 2. have dozens of parameters, many of which are not accurately known and difficult to measure. Additionally, each model dramatically simplifies reality; our parameters are phenomenological summaries of the "true" heterogeneous dynamics. Therefore, we give the user control over all model parameters in order to facilitate the exploration of the dependence of the predicted results on the input parameter values, see Fig. 2 $\mathrm{A}$ for a screenshot of the UI. We note that users specify timescales instead of rates in the UI, e.g. $\gamma_{i}^{-1}$ corresponds to the "Infectious period" input box of Fig. 2 $\mathrm{A}$, as we felt timescales are easier to directly interpret. For ease of use, the web application has presets for many countries and states that can be used as a starting point for exploration.

\section{Interventions}

In order to model both the historical transmission of COVID-19 and project its further spread, one must model the enacted social distancing measures, case isolation, and quarantine policies. As such, our model gives the user the ability to specify individual interventions, indexed by $\alpha$, with a well-defined start and end date and an "effectiveness" $\epsilon_{\alpha} \in[0,1]$ parameter that quantifies the mitigation's multiplicative effect on rate of transmission. See Fig. $2 \mathrm{~B}$ for an depiction of the UI for the input of different mitigation measures. At each point in time, the cumulative efficacy of all interventions is calculated as

$$
\mathcal{M}(t)=\prod_{\alpha \in M(t)}\left(1-\epsilon_{\alpha}\right)
$$

where the product runs over all measures $\mathcal{M}(t)$ in effect at time $t$. In the absence of mitigation strategy, $\mathcal{M}=$ 


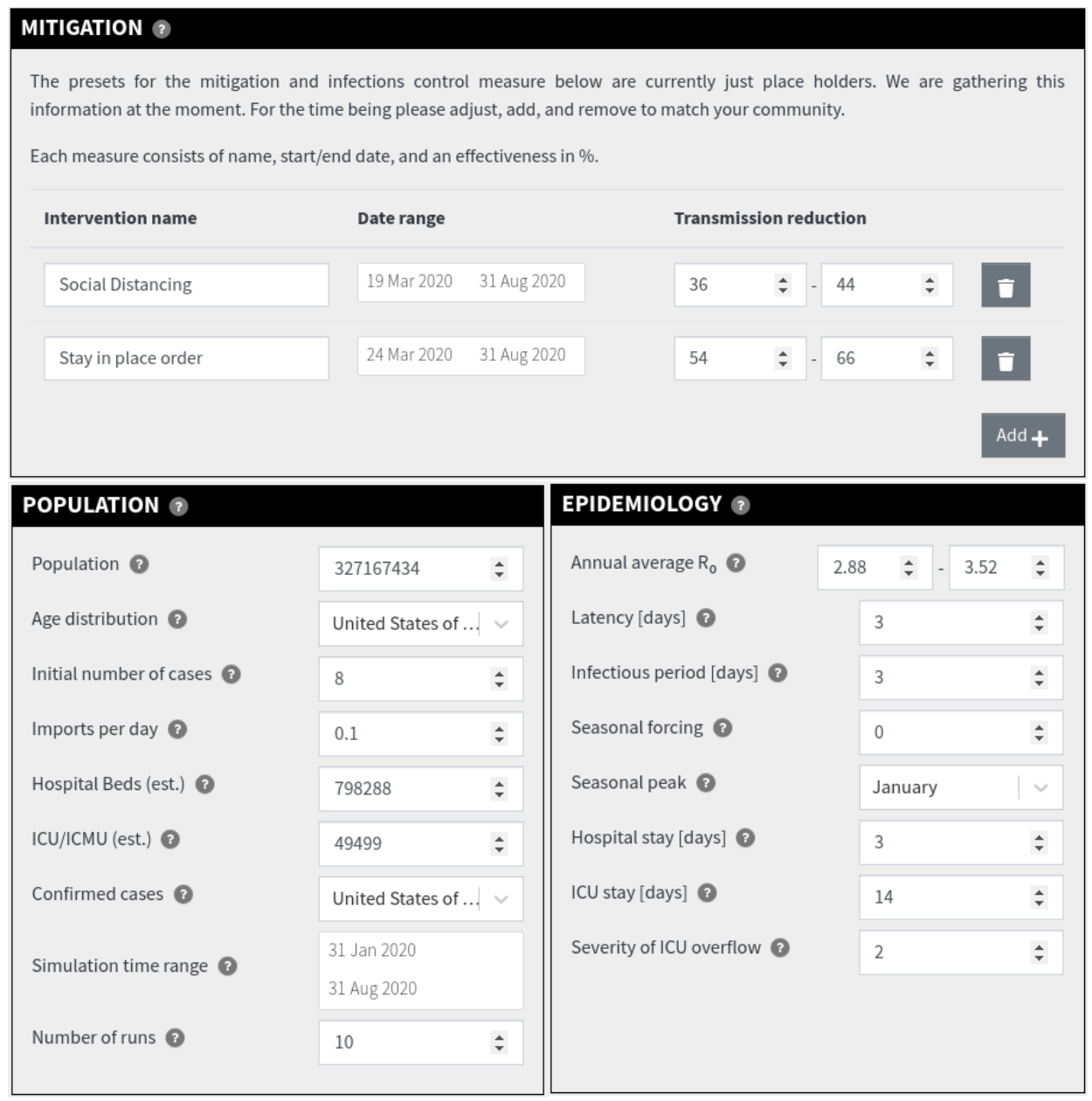

Figure 2 User interface for model parameters and mitigations A) An example screenshot of the UI of the web application used to vary model parameters. On the left, we have grouped parameters of the population under study, e.g. the initial number of cases and the size of population. Conversely, on the right, we grouped phenomenological parameters related to COVID-19 epidemiology. All numbers can be entered manually with a keyboard or stepped with the scrollbox. B) Individual mitigation measures can be added or removed from the model via the the shown interface. Each intervention has a unique name, a range of times it is applied for, and a range of possible efficacies. The net mitigation on COVID-19 transmission is calculated via Eq. 3

1. The overall mitigation efficacy modulates COVID-19 transmissibility as seen in Eqn. 1.

Additionally, our model allows for the input of simple, time-independent age-specific isolation measures. As can be seen in Fig. 3, we provide a column for "age-specific" isolation. These numbers result in a reduction in exposure of individuals from specific age-groups to the general population. For example, this feature could explore the effect of measures specific for the elderly.

\section{Severity parameters and demographics}

The clinical outcome of a COVID-19 infection strongly depends on the age of the patient (Zhou et al., 2020). Hence, the overall burden of a COVID-19 epidemic within a given region strongly depends on the age demo- graphics of the population. In order to facilitate the integration of such effects within the model, we aggregated age distributions for most countries, obtained from the UNSD database API (United Nations Statistics Division, $2020)$ with a custom python script, to provide as presets. Additionally, we allow for custom age distributions to be specified within the UI, see Fig. 3 . The provided age distributions determine the fraction of people in each age group in the simulation.

The Chinese CDC provided extensive statistics of severity of COVID-19 in different age groups (The Novel Coronavirus Pneumonia Emergency Response Epidemiology Team, 2020), broadly compatible with estimates by (Verity et al., 2020). We used these data to parameterize the expected burden on health care systems. Our severity assumptions are summarized in a editable table in the tool, shown in Fig. 3. Each column can be edited 
and changed if users want and the implied infection fatality for each group is calculated. Elements of the table directly correspond to model parameters: (i) $m_{a}$ is the product of the percentage confirmed and the complement of the severe fraction, (ii) $c_{a}$ is set by the critical column, and (iii) $f_{a}$ is set by the fatal fraction.

\section{Parameter uncertainty}

The dynamics of an exponentially growing process such as the COVID-19 pandemic is naturally most sensitive to the growth rate. In the context of the model, the growth rate of infections is primarily a function of the basic reproductive number $R_{0}$ and the societal interventions enacted to slow the spread of COVID-19. Additionally, it is a priori difficult to know the efficacy of mitigation measures. COVID-19 scenarios therefore allows the user to specify ranges for $R_{0}$ as well as for the efficacy $\epsilon_{\alpha}$ of mitigation measures, see Fig. 2 $\mathrm{AB}$ for an example of each. The tool will randomly sample a user-specified number of parameter combinations uniformly from these ranges (by default set to 10 combinations). The outputted results display the median as well as a shaded area denoting the 20th and 80th percentile, see Fig. 4 for an example of the displayed results.

\section{Model results}

The primary result of the tool are trajectories of the number of cases, people in need of hospitalization, and fatalities, see Fig. 4 for an example of the predictions for New York City. Additionally, all predicted trajectories can be exported as a single age-stratified table for further downstream analysis. A short executive summary of the results can additionally be printed to PDF. Where available, the app graphs the recent cases counts, deaths, and hospitalizations for the community under study on the plot with the model results. The surveillance data enables the user to adapt parameters to tune the simulation to the data, see Fig. 4. Once the model fits past data, the user can explore future scenarios by adjusting interventions and seasonality.

\section{ESTIMATION OF PARAMETERS FROM DATA}

While COVID-19 Scenarios is not intended as an inference tool, we nevertheless provide parameter presets that are estimated from empirical data. The primary intent behind fitting to data is not to provide values with high confidence, but rather to facilitate the immediate utility of our tool for different scenarios from across the globe with reasonable presets. We note that care must be taken to not overfit the data; there are many more parameters of the model than features within the available data. Furthermore, the testing and reporting patterns are heterogeneous across regions, as well as change over time which ultimately distort the raw numbers.

We therefore elect to only estimate three model parameters for each region: (i) $R_{0}$, not solely a property of the virus but also the social structure of the population, (ii) the initial date of the epidemic $t_{m i n}$, and (iii) the size of the initial cluster $I_{0}$. In addition, we preset mitigation measures that set in when case-counts rise above certain levels. Again, these are not meant as fit parameters but as templates to be adjusted by the user. We assume the remaining parameters don't vary across regions. These have to be adjusted by the user if the data or other information suggests values different from the defaults.

We try to fit data solely from the onset of the epidemic prior to mitigation efforts from individual regions. Due to the heterogeneity of both the timing and efficacy of policies implemented across the regions provided for interactive exploration, we opted for a simple solution. As more data from more regions become available, we might fit more parameters to observations.

\section{EMPIRICAL DATA}

Both the initial estimates for scenario values, as well as the interactive calibration of the model require empirical observations of COVID-19 infections and hospitalizations. Due to the scope of scenarios provided, we utilize a number of online resources to aggregate information on new COVID-19 cases, deaths, and hospitalizations. These resources include the daily updated case counts by ECDC (European Centre for Disease Control, 2020), the US COVID tracking project (The COVID tracking project, 2020), other official governmental agencies from around the world, and data aggregated by volunteers. A full list of all sources we use can by found in the file data/sources. json in https://github.com/ neherlab/covid19_scenarios. The case empirical data in the app is updated every $2-3$ days.

\section{IMPLEMENTATION AND AVAILABILITY}

COVID-19 Scenarios is implemented as a single-page web application using React web framework, Typescript and numerous packages from Node.js ecosystem. The simulation itself runs on the client side in a WebWorker, to ensure interactivity during the computation. The application can be hosted on any static web-server, or run locally. We host the latest release version publicly on AWS infrastructure, accessible at https:// covid19-scenarios.org.

Data fetching, processing, parameter estimation and scenario generation is implemented using Python and common data science packages, as an additional build 


\begin{tabular}{cc} 
Age group & Age distribution \\
\hline $0-9$ & 884945 \\
$10-19$ & 834866 \\
$20-29$ & 1039727 \\
$30-39$ & 1219227 \\
$40-49$ & 1166590 \\
$50-59$ & 1320623 \\
$60-69$ & 977436 \\
$70-79$ & 751994 \\
$80+$ & 459214 \\
\hline
\end{tabular}

Confirmed
$\%$ total
5
5
10
15
20
25
30
40
50

Severe
\% of confirmed
1
3
3
3
6
10
25
35
50

\begin{tabular}{c|c}
$\begin{array}{c}\text { Critical } \\
\text { \% of severe }\end{array}$ & $\begin{array}{c}\text { Fatal } \\
\text { \% of critical }\end{array}$ \\
\hline 5 & 30 \\
10 & 30 \\
10 & 30 \\
15 & 30 \\
\hline 20 & 30 \\
\hline 25 & 40 \\
\hline 35 & 40 \\
\hline 45 & 50 \\
\hline 55 & 50 \\
\hline
\end{tabular}

$\begin{gathered}\text { Fatal } \\ \% \text { of all infections }\end{gathered}$
0.00075
0.0045
0.009
0.02
0.072
0.25
1.1
3.1
6.9

Isolated
$\%$ total
0
0
0
0
0
0
0
0
0

Figure 3 Age-dependent parameters. Parameters that depend on patient age are summarized in a table that contains the distribution of age groups, severity parameters, and age-specific isolation. The severity parameters are approximately based on data by Surveillances (2020). The first column "confirmed" is our assumption on what fraction of total infections in the different age groups enter as cases in the data analyzed in Surveillances (2020). Young individuals are often asymptomatic and hence less likely to be tested. The following columns specify what fraction of confirmed cases fall severely ill and require medical attention, what fraction of the former fall critically ill and requires intensive care, and lastly hat fraction of critically ill patients die. The implied infection fatality rate is given in the second to last column.

step. The full source code is available under MIT license on GitHub at https://github.com/neherlab/ covid19_scenarios. Instructions on how to run the application are documented there. COVID-19 Scenarios was first released on March 92020 and has been updated consistently since.

\section{DISCUSSION}

Countries, states, and communities across the world have to plan and prepare for the outbreaks and potential reemergence of COVID-19. Many countries have expert research groups that develop tailored models and sophisticated inferences (Kucharski et al. 2020, Neil M Ferguson, 2020) to predict individualized outcomes. However, governments and other public organizations without such availability need a flexible tool that models local outbreaks, explore the effect of interventions, and can compare results to past dynamics in an interactive workflow. This is the gap that COVID-19 Scenarios has and continues to fill. To date, we average roughly 8 thousand page loads per day (we don't track users, but estimated these numbers of from CloudFront usage statistics and the number of requests per page load). These requests come from more than 50 countries, with most visitors coming from the USA, Germany, Switzerland, Russia, Austria, and the UK.

In order to estimate the potential future burden on the health care system, users need flexible ways to adjust demographic parameters in addition to local public health care policies (who gets admitted to the ICU, how long are patients hospitalized). At the same time, sensible defaults are required to provide a useful starting point for exploration. COVID-19 Scenarios was written with the explicit purpose to aid in this regard.

The past few months have shown that social distancing measures can effectively slow the spread of COVID-
19(Wang et al. 2020$)$. The future trajectory of COVID19 will therefore primarily depend upon the level of social distancing and infection control that is maintained. COVID-19 Scenarios therefore cannot confidently predict outcomes, but rather help to explore potential future scenarios under specific assumptions made by the user. This difficulty is further compounded by the fact that the prediction of absolute numbers are exceedingly sensitive to small variations in input parameters. Due to nature of exponential growth experienced within an epidemic, a small uncertainty in either the growth rate or initial date will naturally result in large uncertainty in case numbers. Therefore, it is critical that these uncertainties are communicated effectively to policy makers. We therefore allow the user to specify plausible ranges for the parameter $R_{0}$ and the efficacy of the interventions. In case of several interventions, this results in a high dimensional space of possibilities that we sample uniformly. Percentiles of the sampled results are displayed to capture the range of potential outcomes.

We stress that in addition to parameter uncertainty, a simple SEIR model is a drastic abstraction and simplification that does not capture the full complexity and heterogeneity of the outbreak. Nevertheless we hope that the tool is helpful for understanding the dynamic of the outbreak and exploring the effect of past and future interventions.

\section{ACKNOWLEDGEMENT}

We gratefully acknowledge input from members of the lab, Adam Kucharski, Rosalind Eggo, and Christian Althaus. Nils Ole Tippenhauer and Aitana Lebrand have helped to parse, aggregate, and update surveillance data from various sources. In addition, we received many invaluable contributions from the open source community. vercel.com has supported the development with free ac- 
medRxiv preprint doi: https://doi.org/10.1101/2020.05.05.20091363; this version posted May 12, 2020. The copyright holder for this preprint (which was not certified by peer review) is the author/funder, who has granted medRxiv a license to display the preprint in perpetuity. It is made available under a CC-BY-NC 4.0 International license.
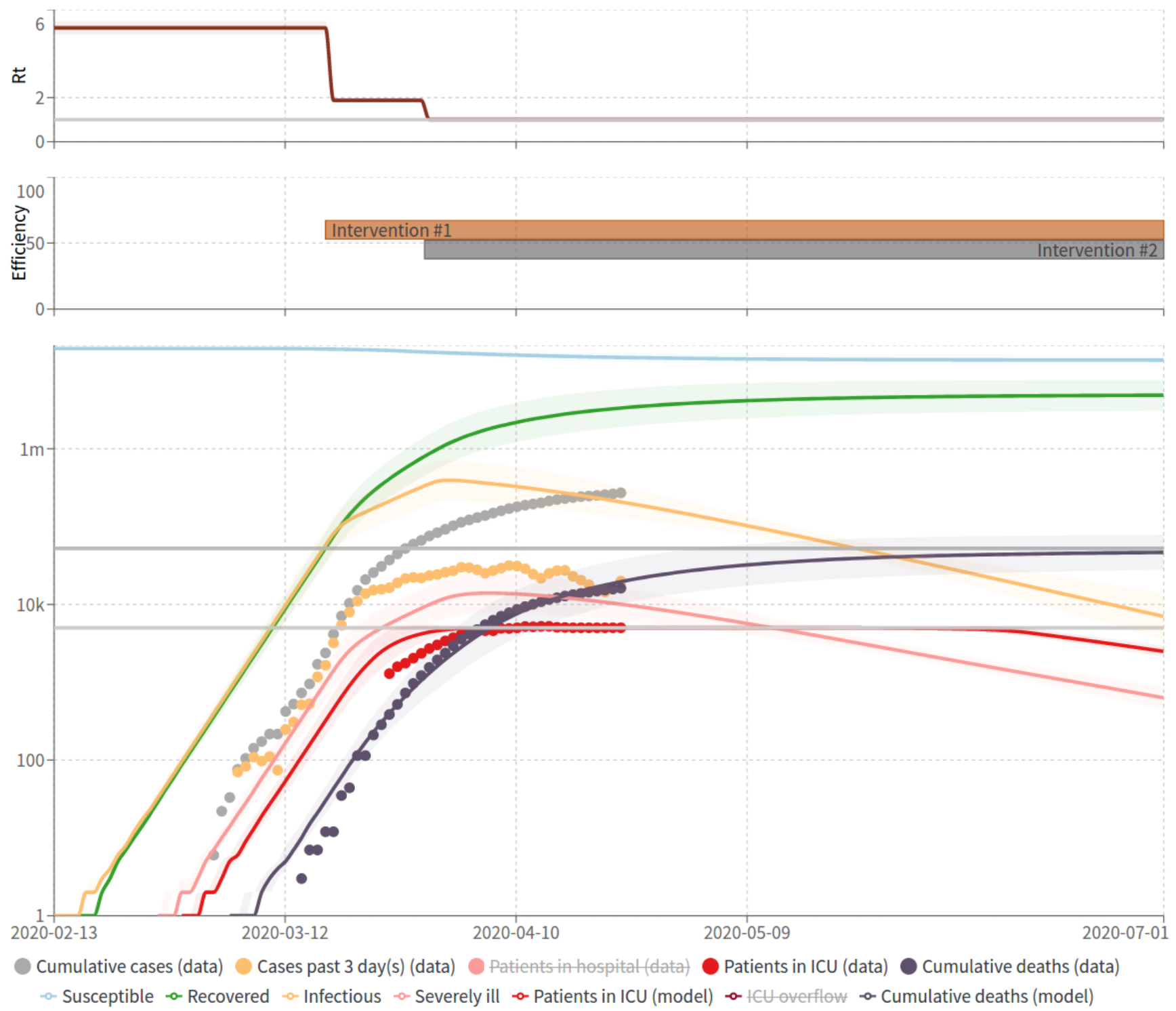

Figure 4 Example simulation results for New York City. (top) A plot of the time-dependent effective reproductive number. This is controlled via mitigation interventions and seasonality. The colored line corresponds to the median and the shaded area is bounded by the $20 \%$ and $80 \%$ percentiles. (middle) Plot that shows both the interval (length) and ranges of possible efficacies (width) for the applied mitigation interventions. (bottom) Plot that shows the resulting trajectories of all compartments. The colored line for each compartment shows the median trajectory while the shaded area is bounded by the $20 \%$ and $80 \%$ percentiles. Additionally, the aggregated case count data is plotted (where available) as individual points. The display of individual compartments can be toggled by clicking on the legend.

cess to their tool-stack.

\section{REFERENCES}

Callow, K A, H. F. Parry, M. Sergeant, and D. A. Tyrrell (1990), "The time course of the immune response to experimental coronavirus infection of man," Epidemiology and Infection 105 (2), 435-446.

European Centre for Disease Control, (2020), "COVID-19,"

Fang, Lei, George Karakiulakis, and Michael Roth (2020),

"Are patients with hypertension and diabetes mellitus at increased risk for COVID-19 infection?" The Lancet. Respiratory Medicine.

Ganyani, Tapiwa, Cecile Kremer, Dongxuan Chen, Andrea Torneri, Christel Faes, Jacco Wallinga, and Niel Hens (2020), "Estimating the generation interval for COVID-19 based on symptom onset data," medRxiv.

"IMHE COVID-19 forecasting team",, and Christopher JL Murray (2020), "Forecasting covid-19 impact on hospital bed-days, icu-days, ventilatordays and deaths by us state in the next 4 months," medRxiv 10.1101/2020.03.27.20043752, https://www.medrxiv.org/content/early/2020/03/30/2020.03.27.20043 
Kermack, William Ogilvy, A. G. McKendrick, and Gilbert Thomas Walker (1927), "A contribution to the mathematical theory of epidemics," Proceedings of the Royal Society of London. Series A, Containing Papers of a Mathematical and Physical Character 115 (772), 700721, publisher: Royal Society.

Kucharski, Adam J, Petra Klepac, Andrew Conlan, Stephen M. Kissler, Maria Tang, Hannah Fry, Julia Gog, John Edmunds, and CMMID COVID-19 Working Group (2020), "Effectiveness of isolation, testing, contact tracing and physical distancing on reducing transmission of SARS-CoV-2 in different settings," medRxiv , 2020.04.23.20077024Publisher: Cold Spring Harbor Laboratory Press.

Lauer, Stephen A, Kyra H Grantz, Qifang Bi, Forrest K Jones, Qulu Zheng, Hannah R Meredith, Andrew S Azman, Nicholas G Reich, and Justin Lessler (2020), "The incubation period of coronavirus disease 2019 (COVID-19) from publicly reported confirmed cases: estimation and application," Annals of internal medicine.

Li, Qun, Xuhua Guan, Peng Wu, Xiaoye Wang, Lei Zhou, Yeqing Tong, Ruiqi Ren, Kathy SM Leung, Eric HY Lau, Jessica Y Wong, et al. (2020), "Early transmission dynamics in Wuhan, China, of novel coronavirus-infected pneumonia," New England Journal of Medicine.

Neher, Richard A, Robert Dyrdak, Valentin Druelle, Emma B Hodcroft, and Jan Albert (2020), "Potential impact of seasonal forcing on a SARS-CoV-2 pandemic," Swiss Medical Weekly 150 (1112).

Neil M Ferguson, Daniel Laydon, Gemma Nedjati-Gilani Natsuko Imai Kylie Ainslie Marc Baguelin Sangeeta Bhatia Adhiratha Boonyasiri Zulma Cucunuba Gina CuomoDannenburg Amy Dighe Ilaria Dorigatti Han Fu Katy Gaythorpe Will Green Arran Hamlet Wes Hinsley Lucy C Okell Sabine van Elsland Hayley Thompson Robert Verity Erik Volz Haowei Wang Yuanrong Wang Patrick GT Walker Caroline Walters Peter Winskill Charles Whittaker Christl A Donnelly Steven Riley Azra C Ghani (2020), "Report 9 - Impact of non-pharmaceutical interventions (NPIs) to reduce COVID-19 mortality and healthcare demand," Library Catalog: www.imperial.ac.uk.

Nishiura, Hiroshi, Natalie M Linton, and Andrei R Akhmetzhanov (2020), "Serial interval of novel coronavirus (COVID-19) infections," International journal of infectious diseases.

Riou, Julien, and Christian L Althaus (2020), "Pattern of early human-to-human transmission of Wuhan 2019 novel coronavirus (2019-ncov), December 2019 to January 2020," Eurosurveillance 25 (4), 2000058.

Surveillances, Vital (2020), "The epidemiological characteristics of an outbreak of 2019 novel coronavirus diseases (COVID-19) - China, 2020," China CDC Weekly 2 (8), 113-122.

The COVID tracking project, (2020), Library Catalog: covidtracking.com.

The Novel Coronavirus Pneumonia Emergency Response Epidemiology Team, (2020), "The Epidemiological Characteristics of an Outbreak of 2019 Novel Coronavirus Diseases (COVID-19) - China, 2020," China CDC Weekly 2 (8), 113-122, publisher: China CDC Weekly.

The WHO COVID-19 group, (2020), "Coronavirus disease 2019 (COVID-19) Situation Report - 51," П.

United Nations Statistics Division, (2020), "World population prospects," Data retrieved from population demographic total datasets using Python pandas interface.

Verity, Robert, Lucy C Okell, Ilaria Dorigatti, Peter Winskill, Charles Whittaker, Natsuko Imai, Gina CuomoDannenburg, Hayley Thompson, Patrick GT Walker, Han $\mathrm{Fu}$, et al. (2020), "Estimates of the severity of coronavirus disease 2019: a model-based analysis," The Lancet Infectious Diseases 10.1016/S1473-3099(20)30243-7

Wang, Chaolong, Li Liu, Xingjie Hao, Huan Guo, Qi Wang, Jiao Huang, $\mathrm{Na} \mathrm{He}$, Hongjie $\mathrm{Yu}$, Xihong Lin, An Pan, Sheng Wei, and Tangchun Wu (2020), "Evolving Epidemiology and Impact of Non-pharmaceutical Interventions on the Outbreak of Coronavirus Disease 2019 in Wuhan, China," medRxiv , 2020.03.03.20030593Publisher: Cold Spring Harbor Laboratory Press.

Zhang, Sheng, MengYuan Diao, Wenbo Yu, Lei Pei, Zhaofen Lin, and Dechang Chen (2020), "Estimation of the reproductive number of novel coronavirus (COVID-19) and the probable outbreak size on the diamond princess cruise ship: A data-driven analysis," International Journal of Infectious Diseases 93, 201-204.

Zhou, Fei, Ting Yu, Ronghui Du, Guohui Fan, Ying Liu, Zhibo Liu, Jie Xiang, Yeming Wang, Bin Song, Xiaoying $\mathrm{Gu}$, et al. (2020), "Clinical course and risk factors for mortality of adult inpatients with COVID-19 in Wuhan, China: a retrospective cohort study," The Lancet. 